\title{
Compacto transitividad y su relación con tipos de sensitividad
}

| Dynamical compactness and its relationship among several types of sensitivity |

\author{
D Irma León-Torres \\ yooirma@gmail.com \\ Universidad Autónoma de San Luis Potosí \\ San Luis Potosí, México
}

\author{
Alicia Santiago-Santos \\ alicia@mixteco.utm.mx \\ Universidad Tecnológica de la Mixteca \\ Huajuapan de León, Oaxaca, México
}

Recibido: 17 agosto 2021

Aceptado: 6 diciembre 2021

Resumen: El objetivo de este artículo es proporcionar al lector una introducción a ciertas clases de sistemas dinámicos. A saber, sensitivos, multi-sensitivo, cofinitamente sensitivo, thick sensitivo, sindéticamente sensitivo, thickly sindéticamente sensitivo. Además, presentar un estudio y analizar la relación que existe entre los tipos de sistemas mencionados con anterioridad y los sistemas dinámicos compacto transitivo introducidos en [8].

Palabras Clave: Sensibilidad, sistema dinámico, caos, cofinitamente sensitivo, multisensitividad.

Abstract: The objective of this article is to provide the reader an introduction to some kinds of dynamical systems. Namely, sensitive systems, multi-sensitive, cofinite sensitive, thickly sensitive, syndetically sensitive, thickly syndetically sensitive. We also present a study and analyzed the relationship between the types of system mentioned above and the dynamical systems transitive compact introduced in [8].

Keywords: Sensitivity, dynamic system, chaos, cofinitely sensitive, multi-sensitivity.

\section{Introducción}

El presente trabajo se desarrolla en el área de la matemática denominada Dinámica topológica. En este artículo los sistemas dinámicos que estudiaremos son los sistemas dinámicos discretos. De manera abstracta, un sistema dinámico discreto será una pareja $(X, f)$, donde $X$ es un espacio métrico compacto, conexo con más de un elemento y $f: X \rightarrow X$ es una función continua y suprayectiva. En la actualidad, se han desarrollado algunas publicaciones, por mencionar, vea por ejemplo $[3,4,5,15]$.

Hasta ahora, aún no hay una definición matemática que se acepte universalmente para el caos, sin embargo se sabe que dos de las nociones para entender un sistema caótico son la transitividad y la sensitividad [10]. Por tal motivo, a través del tiempo, han surgido nociones relacionadas con la transitividad y nociones relacionadas con la sensibilidad. En 1971, Ruelle introdujo la primera definición de sensitividad [16]. De forma intuitiva, un sistema dinámico es sensitivo si arbitrariamente cerca a 
cualquier punto de inicio existe al menos otro punto tal que alguna de sus iteraciones bajo la función se aleja de la iteración correspondiente del punto con el que se inició. Dada la importancia de los sistemas dinámicos sensitivos, empezaron a surgir otras nociones relacionadas con la sensitividad, como: sensitividad Li-Yorke [1], sensitividad thick sindética [13], sensitividad sindética [17], sensitividad cofinita [17] y multi-sensitividad [17]. Dada la relevancia y utilidad de los sistemas transitivos y sensitivos para tratar de entender el caos, se buscan nociones relacionadas con este tipo de sistemas. Recientemente, en 2016, W. Huang, D. Khilko, S. Kolyada y G. Zhang en [8], estudian un tipo de sistemas dinámicos transitivos, a saber, los sistemas dinámicos compacto transitivos; analizan la relación de estos nuevos sistemas con los sistemas del tipo transitivos y con los sistemas del tipo sensitivos.

En este trabajo nos enfocamos en analizar varios conceptos relacionados con la sensibilidad sobre un sistema dinámico discreto y a ver su relación entre estos conceptos, este trabajo está basado en [11]. Cabe mencionar que aunque los resultados expuestos en este escrito son conocidos este posee originalidad en cuanto a su escritura y presentación la cual se hace en forma detallada, con la finalidad dé lograr su accesibilidad a un mayor número de lectores qué se interesen en estos temas. Por este motivo, este trabajo está dividido en seis secciones. Después de la introducción, en la sección 2, exponemos algunos conceptos y notaciones que se utilizan durante el escrito. La sección 3 está dedicada al análisis de propiedades generales de los conjuntos cofinito, thick, sindético, etc. En la sección 4, se describen las relaciones entre los sistemas dinámicos que estamos estudiando. La sección 5 trata sobre los sistemas compacto transitivos. Finalmente, en la sección 6 se incluye la relación entre los sistemas compacto transitivos y sistemas de tipo sensitivos.

\section{Preliminares}

En esta sección presentamos los conceptos básicos que se utilizan durante el desarrollo de este trabajo. También introducimos la notación y terminología que usaremos frecuentemente. Como es usual, denotaremos por $\mathbb{N}, \mathbb{R}, \mathbb{Z}, \mathbb{Z}_{+}$, al conjunto de los números naturales, los números reales, los números enteros y los números enteros no negativos, respectivamente. Ahora, si $X$ es un espacio métrico y $f: X \rightarrow X$ es una función, establecemos la siguiente notación: $f^{0}$ denotará la función identidad sobre $X, i d_{X}$, para cada $n \in \mathbb{N}, f^{n}$ es $f$ compuesta con $f^{n-1}$, es decir:

$$
f^{n}=f \circ f^{n-1} .
$$

Además, dados $n \in \mathbb{N}$ y $A \subseteq X$, la preimagen de $A$ bajo $f^{n}$ se indica por $f^{-n}(A)$ y si $x \in X$, la preimagen de $\{x\}$ bajo $f$ la representamos por $f^{-1}(x)$. Si $A \subseteq X$ no vacío y acotado, se define el diámetro de $A$, denotado por diám $(A)$, como:

$$
\operatorname{diám}(A)=\sup \{d(x, y): x, y \in A\} \text {. }
$$

A continuación presentamos uno de los conceptos importantes en este trabajo.

\section{Definición 1}

Un sistema dinámico discreto es una pareja $(X, f)$, donde $X$ es un espacio métrico compacto, conexo, con más de un elemento y $f: X \rightarrow X$ una función continua y suprayectiva.

En este trabajo para referirnos a un sistema dinámico discreto diremos solamente sistema dinámico. Se sabe que el objeto principal de estudio de los sistemas dinámicos discretos son las órbitas de los puntos. En seguida presentamos dicho concepto. 


\section{Definición 2}

Sean $(X, f)$ un sistema dinámico y $x \in X$. Se define la órbita de $x$ bajo $f$, denotada por $\mathcal{O}(x, f)$, como el conjunto:

$$
\mathcal{O}(x, f)=\left\{f^{n}(x): n \in \mathbb{Z}_{+}\right\}=\left\{x, f(x), f^{2}(x), f^{3}(x), \ldots\right\} .
$$

La interpretación que se le da a el conjunto $\mathcal{O}(x, f)$ es la siguiente: En el tiempo $n=0$ un objeto se encuentra en la posición $x$; en el tiempo $n=1$ el objeto ahora a cambiado de posición y se encuentra en la posición $f(x)$; en el tiempo $n=2$, el objeto nuevamente a cambiado de posición y ahora se encuentra en $f^{2}(x)$, etc.

En seguida presentamos algunos tipos de puntos asociados con $f$.

\section{Definición 3}

Sean $(X, f)$ un sistema dinámico y $x \in X$. Decimos que $x$ es un:

1. Punto fijo si $f(x)=x$.

2. Punto periódico si existe $n \in \mathbb{N}$ tal que $f^{n}(x)=x$, al $\operatorname{mín}\left\{n \in \mathbb{N}: f^{n}(x)=x\right\}$ se le llama periodo de $x$.

3. Punto transitivo si $\mathcal{O}(x, f)$ es un conjunto denso en $X$.

4. Punto casi-periódico si para cada vecindad $U_{x}$ de $x$, existe $k \in \mathbb{N}$ tal que

$$
\left\{f^{l}(x), f^{l+1}(x), \ldots, f^{l+k}(x)\right\} \cap U_{x} \neq \emptyset,
$$

para todo $l \in \mathbb{N}$.

5. Punto pre-periódico si existen $n, k \in \mathbb{N}$, tales que $f^{k}\left(f^{n}(x)\right)=f^{n}(x)$.

Denotamos como $\operatorname{Per}(f), \operatorname{Fix}(f)$ y $\operatorname{Tran}(X, f)$, al conjunto de los puntos periódicos de $f$, al conjunto de puntos fijos de $f$ y al conjunto de los puntos transitivos de $f$, respectivamente.

En la Figura 1 se muestra geométricamente el comportamiento de un punto fijo, un punto periódico y un punto pre-periódico.

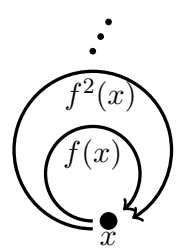

(a) Punto fijo

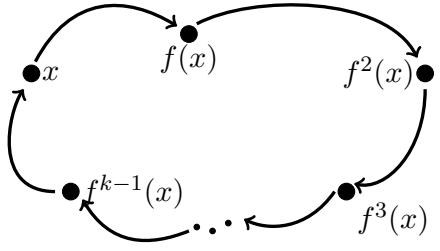

(b) Punto periódico

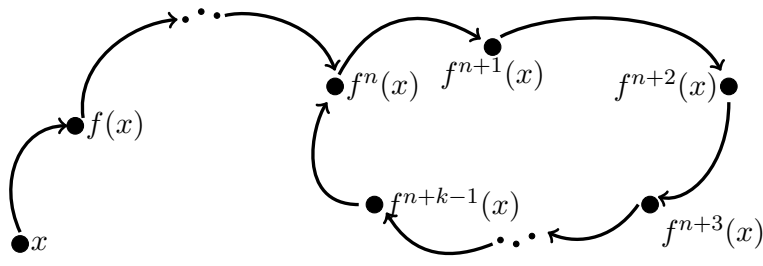

(c) Punto pre-periódico

Figura 1: Comportamiento geométrico.

\section{Observación 1}

Sean $A \subseteq \mathbb{R}$ y $f: A \rightarrow A$ una función continua. Para obtener geométricamente los puntos fijos de $f$ se grafica la función $f$ y la función identidad en $A$. Los puntos de intersección de ambas gráficas son los puntos fijos de $f$.

En la dinámica topológica, existen funciones que determinan sistemas dinámicos muy interesantes y que ayudan a entender de alguna manera el comportamiento que se puede generar, además tales 
funciones son útiles para comprender la dinámica de otras funciones más complicadas. Uno de los ejemplos clásicos de sistemas dinámicos discretos es la función tienda.

\section{Ejemplo 1}

La función tienda, $T:[0,1] \rightarrow[0,1]$, está definida como:

$$
T(x)= \begin{cases}2 x, & \text { si } \quad x \in\left[0, \frac{1}{2}\right] ; \\ 2-2 x, & \text { si } x \in\left[\frac{1}{2}, 1\right],\end{cases}
$$

para cada $x \in[0,1]$. En la Figura 2 se muestra geométricamente la gráfica de dicha función.

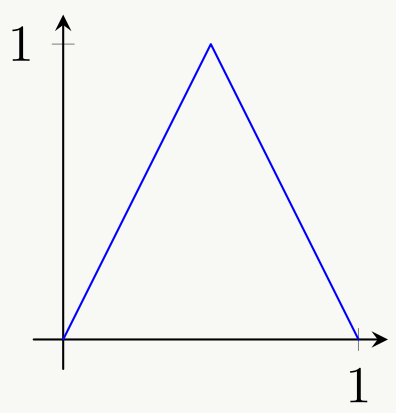

Figura 2: Gráfica de la función tienda.

Obtengamos los puntos fijos de la función $T$, es decir, los puntos $x \in[0,1]$ tales que $T(x)=x$. Para eso consideremos los siguientes casos:

Caso 1. $x \in\left[0, \frac{1}{2}\right]$. En este caso, $T(x)=2 x$. Luego, $2 x=x$ esto implica que $x=0$.

Caso 2. $x \in\left[\frac{1}{2}, 1\right]$. En este caso $T(x)=2-2 x$. Luego, $2-2 x=x$, esto implica que $2=3 x$, de donde $x=\frac{2}{3}$.

Por lo tanto, los puntos fijos de la función $T$, son $x=0$ y $x=\frac{2}{3}$. En la Figura 3 presentamos geométricamente los puntos fijos de la función.

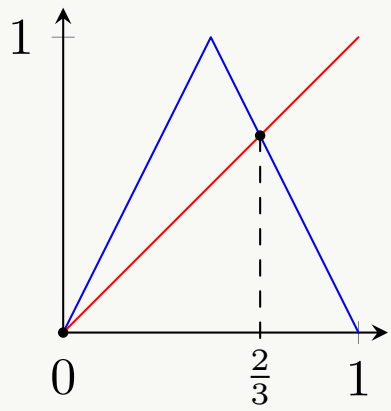

Figura 3: Puntos fijos de la función tienda.

A continuación definimos algunos sistemas dinámicos conocidos en esta área de la matemática y de nuestro interés.

\section{Definición 4}

Sea $(X, f)$ un sistema dinámico discreto. Se dice que $(X, f)$ es:

1. Exacto si para cada subconjunto abierto no vacío $U$ de $X$, existe $k \in \mathbb{N}$ tal que $f^{k}(U)=X$. 
2. Mezclante si para cualesquiera dos subconjuntos abiertos, no vacíos $U, V$ de $X$, existe $N \in$ $\mathbb{N}$ tal que $f^{k}(U) \cap V \neq \emptyset$, para todo $k \geq N$.

3. Débilmente mezclante si para cualesquiera $U_{1}, U_{2}, V_{1}$ y $V_{2}$ subconjuntos abiertos no vacíos de $X$, existe $n \in \mathbb{N}$ tal que $U_{1} \cap f^{-n}\left(V_{1}\right) \neq \emptyset$ y $U_{2} \cap f^{-n}\left(V_{2}\right) \neq \emptyset$.

4. Transitivo si para cualesquiera dos subconjuntos $U, V$ abiertos no vacíos de $X$ existe $n \in \mathbb{N}$ tal que $U \cap f^{-n}(V) \neq \emptyset$.

5. M-sistema si el sistema es transitivo y el conjunto de los puntos casi-periódicos es denso en $X$.

6. Caótico si $(X, f)$ es transitivo y $\operatorname{Per}(f)$ es denso en $X$.

En seguida mostramos algunas propiedades de la función tienda, las cuales están planteadas en [14, pág. 39].

\section{Ejemplo 2}

Consideremos el sistema dinámico $([0,1], T)$ donde $T$ se definió en el Ejemplo 1. Se cumple que:

1. $([0,1], T)$ es exacto;

2. $([0,1], T)$ es mezclante y

3. $([0,1], T)$ es caótico.

Para poder definir más tipos de sistemas dinámicos discretos introducimos los siguientes conjuntos.

\section{Definición 5}

Sean $(X, f)$ un sistema dinámico, $x \in X, U$ y $V$ subconjuntos abiertos no vacíos de $X$ y $\delta>0$. Se definen los siguientes conjuntos:

1. $N_{f}(x, V)=\left\{n \in \mathbb{Z}_{+}: f^{n}(x) \in V\right\}$.

2. $N_{f}(U, V)=\left\{n \in \mathbb{Z}_{+}: f^{n}(U) \cap V \neq \emptyset\right\}$.

3. $N_{f}(U, \delta)=\left\{n \in \mathbb{Z}_{+}\right.$: existen $x, y \in U$ tales que $\left.d\left(f^{n}(x), f^{n}(y)\right)>\delta\right\}$.

A continuación enunciamos algunas observaciones que se obtienen inmediatamente de la Definición 5 .

\section{Observación 2}

Sean $(X, f)$ un sistema dinámico, $U, U_{1}, V$ y $V_{1}$, subconjuntos abiertos no vacíos de $X$ y $\delta>0$.

1. Si $U \subseteq V$, entonces $N_{f}(U, \delta) \subseteq N_{f}(V, \delta)$.

2. Si $U \subseteq U_{1}$ y $V \subseteq V_{1}$, entonces $N_{f}(U, V) \subseteq N_{f}\left(U_{1}, V_{1}\right)$.

En seguida, se muestra un ejemplo de los conjuntos dados en la Definición 5. 


\section{Ejemplo 3}

Sean $(X, f)$ un sistema dinámico discreto, $x \in X, U$ y $V$ subconjuntos abiertos no vacíos de $X$ y $\delta>0$. Suponiendo que la órbita de $x$ tiene un comportamiento como el que se muestra en la Figura 4 , se obtiene que $\{3,4,6\} \subseteq N_{f}(x, V)$. Por otro lado, de la Figura 5 se visualiza el conjunto $N_{f}(U, V)$. Supongamos que las iteraciones de $U$ se comportan como en la Figura 5 . Por lo tanto, podemos concluir que al menos $\{2,3\} \subseteq N_{f}(U, V)$. Finalmente, de la Figura 6 se observa que $2 \in N_{f}(U, \delta)$, pues existen $x$ y $y$ en el conjunto $U$ tales que $d\left(f^{2}(x), f^{2}(y)\right)>\delta$.

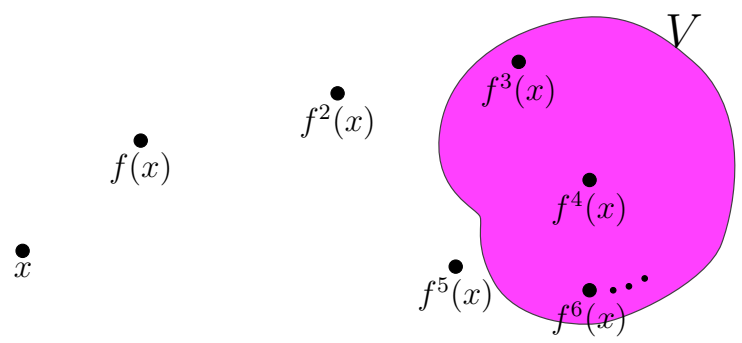

Figura 4: Diagrama para obtener el conjunto $N_{f}(x, V)$

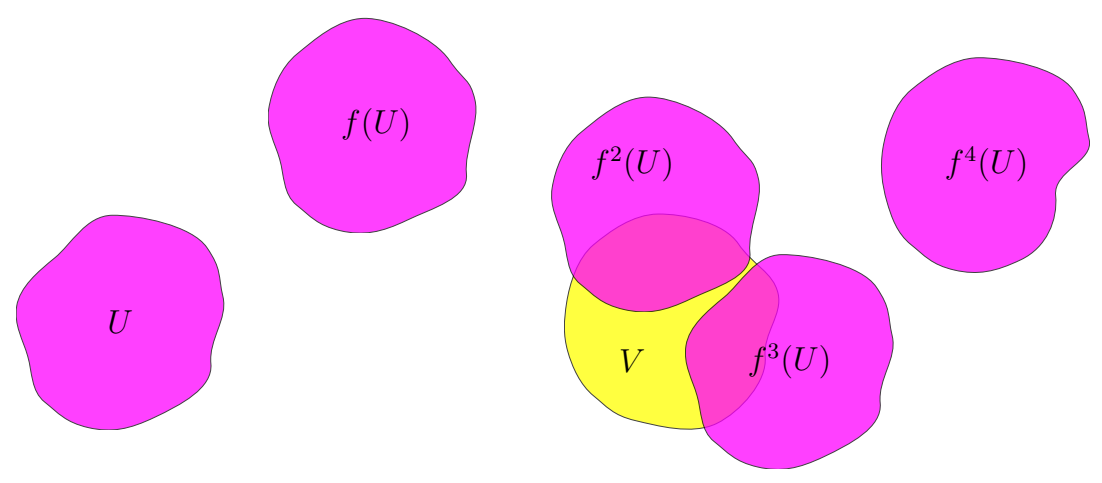

Figura 5: Diagrama para obtener el conjunto $N_{f}(U, V)$.
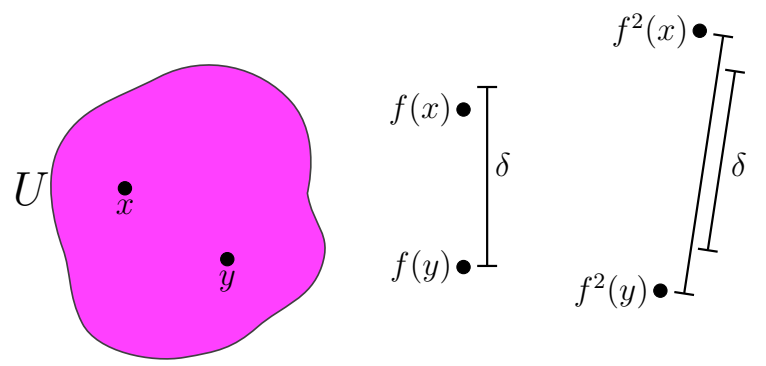

Figura 6: Diagrama para obtener el conjunto $N_{f}(U, \delta)$.

El siguiente resultado, Proposición 1, nos será útil para mostrar otro ejemplo del comportamiento de los conjuntos presentados en la Definición 5.

\section{Proposición 1}

Sean $(X, f)$ un sistema dinámico, $U$ y $V$ subconjuntos no vacíos de $X$ y $n \in \mathbb{N}$. Se cumple que $U \cap f^{-n}(V) \neq \emptyset$ si y solo si $f^{n}(U) \cap V \neq \emptyset$.

Demostración. Supongamos que $U \cap f^{-n}(V) \neq \emptyset$. Luego, existe $x \in X$ tal que $x \in U$ y $x \in f^{-n}(V)$. Así, $f^{n}(x) \in f^{n}(U)$. Esto implica que $f^{n}(x) \in V$. Por lo tanto, $f^{n}(U) \cap V \neq \emptyset$. Ahora, supongamos que 
$f^{n}(U) \cap V \neq \emptyset$. Luego, existe $y \in X$ tal que $y \in f^{n}(U)$ y $y \in V$. Es decir, existe $x \in U$ tal que $f^{n}(x)=y$. Luego, $f^{n}(x) \in V$. Así, $x \in f^{-n}(V)$. Por lo tanto, $U \cap f^{-n}(V) \neq \emptyset$.

En el Ejemplo 4, con la ayuda de la Proposición 1, calculamos los conjuntos dados en la Definición 5 para un sistema dinámico en particular.

\section{Ejemplo 4}

Consideremos el intervalo cerrado $[0,1]$ con la métrica del subespacio de la recta real y el sistema dinámico $([-1,1], f)$, donde $f$ está definida por $f(x)=x^{3}$, para cada $x \in[-1,1]$.

Sean $x_{0}=\frac{1}{2}$ y $W=\left(\frac{1}{3}, \frac{4}{5}\right)$. Determinemos $N_{f}\left(x_{0}, W\right)$. Notemos que:

$f^{0}\left(\frac{1}{2}\right)=\frac{1}{2}, \quad f^{1}\left(\frac{1}{2}\right)=\left(\frac{1}{2}\right)^{3}=\frac{1}{8}, \quad f^{2}\left(\frac{1}{2}\right)=f\left(f\left(\frac{1}{2}\right)\right)=f\left(\frac{1}{8}\right)=\left(\frac{1}{8}\right)^{3}=\frac{1}{8^{3}}, \quad \ldots$

Así $\frac{1}{8}, \frac{1}{8^{3}} \notin W$. Luego, $1,2 \notin N_{f}\left(x_{0}, W\right)$. De esta manera se puede notar que $\mathbb{N} \nsubseteq N_{f}\left(x_{0}, W\right)$. Por lo tanto, $N_{f}\left(x_{0}, W\right)=\{0\}$. Ahora, consideremos los subconjuntos $U=\left(\frac{1}{4}, \frac{1}{2}\right)$ y $V=\left(-\frac{1}{2}, \frac{1}{2}\right)$ de $[-1,1]$. Determinemos $N_{f}(U, V)$. Observemos que:

$$
\begin{aligned}
f^{0}(U)=\left(\frac{1}{4}, \frac{1}{2}\right) & f^{2}(U)=f(f(U))=f\left(U^{3}\right)=\left(U^{3}\right)^{3}=\left(\frac{1}{4^{9}}, \frac{1}{2^{9}}\right) \\
f^{1}(U)=U^{3}=\left(\frac{1}{4^{3}}, \frac{1}{2^{3}}\right) & f^{3}(U)=f\left(f^{2}(U)\right)=f\left(U^{9}\right)=\left(U^{9}\right)^{3}=\left(\frac{1}{4^{27}}, \frac{1}{2^{27}}\right)
\end{aligned}
$$

Luego, $f^{0}(U) \cap V \neq \emptyset, f^{1}(U) \cap V \neq \emptyset, f^{2}(U) \cap V \neq \emptyset$ y $f^{3}(U) \cap V \neq \emptyset$. En consecuencia $N_{f}(U, V)=$ $\{0,1,2,3, \ldots\}$.

\section{Proposición 2}

Sean $(X, f)$ un sistema dinámico, $U$ subconjunto abierto no vacío en $X, n, m \in \mathbb{Z}_{+} \mathrm{y} \delta>0$. Si $m+n \in N_{f}\left(f^{-n}(U), \delta\right)$, entonces $m \in N_{f}(U, \delta)$.

Demostración. Supongamos que $n+m \in N_{f}\left(f^{-n}(U), \delta\right)$. Esto implica que existen $x, y \in f^{-n}(U)$ tales que:

$$
d\left(f^{m+n}(x), f^{m+n}(y)\right)>\delta
$$

equivalentemente,

$$
d\left(f^{m}\left(f^{n}(x)\right), f^{m}\left(f^{n}(y)\right)\right)>\delta
$$

Por otro lado, como $x, y \in f^{-n}(U)$ se tiene que $f^{n}(x), f^{n}(y) \in U$. Por lo tanto, de (1), se concluye que $m \in N_{f}(U, \delta)$. 


\section{Propiedades en general}

En esta sección presentaremos algunas relaciones que se tienen entre los conjuntos thick, cofinito, sindético y thickly sindético, conceptos necesarios para definir más clases de sistemas dinámicos.

\section{Definición 6}

Sea $A \subseteq \mathbb{Z}_{+}$. Se dice que el conjunto $A$ es:

1. Cofinito si existe $k \in \mathbb{N}$ tal que $\{k, k+1, k+2 \ldots\} \subseteq A$.

2. Thick si para cada $p \in \mathbb{N}$, existe $n \in \mathbb{N}$ tal que $\{n, n+1, \ldots, n+p\} \subseteq A$.

3. Sindético si existe $m \in \mathbb{N}$ tal que para todo $l \in \mathbb{N}$ se cumple que $\{l, l+1, \ldots, l+m\} \cap A \neq \emptyset$.

4. Thickly sindético si para cada $n \in \mathbb{N},\{i \in \mathbb{N}:\{i, i+1, \ldots, i+n\} \subseteq A\}$ es sindético.

En el Ejemplo 5 se muestran algunos ejemplos de los conjuntos dados en la Definición 6.

\section{Ejemplo 5}

Sean $m \in \mathbb{Z}_{+}$y $B \subseteq \mathbb{Z}_{+}$. Se cumple que el conjunto:

1. $A=\{n \in \mathbb{N}: n \geq m\}$ es cofinito.

2. $A=\{n \in \mathbb{N}: n$ no es divisor de $m\}$ es cofinito.

3. Si $B$ es finito, entonces el conjunto $A=\mathbb{N} \backslash B$ es cofinito.

4. $A=\{n \in \mathbb{N}: n$ es un número par $\}$ es sindético.

5. $A=\{n \in \mathbb{N}: n$ es un número impar $\}$ es sindético.

6. $A=\{n \in \mathbb{N}: n$ es un múltiplo de $m\}$ es sindético.

Los conjuntos dados en la Definición 6 poseen propiedades interesantes. Una prueba de los siguientes resultados, Proposición 3 y Proposición 4, puede encontrarla en [12].

\section{Proposición 3}

Sean $A$ y $B$ subconjuntos de $\mathbb{Z}_{+}$tales que $A \subseteq B$. Si $A$ es thick, entonces $B$ es thick.

\section{Proposición 4}

Sea $A \subseteq \mathbb{Z}_{+}$. Si $A$ es cofinito, entonces $A$ es thick.

\section{Proposición 5}

Sea $A \subseteq \mathbb{Z}_{+}$. Si $A$ es cofinito, entonces $A$ es thickly sindético.

Demostración. Supongamos que $A$ es cofinito. Demostremos que $A$ es thickly sindético. Sea $n \in \mathbb{N}$. Veamos que el conjunto $\{i \in \mathbb{N}:\{i, i+1, \ldots, i+n\} \subseteq A\}$ es sindético. Por hipótesis, existe $k \in \mathbb{N}$ tal que

$$
\{k, k+1, k+2, \ldots\} \subseteq A .
$$


Sea $l \in \mathbb{N}$. Veamos que:

$$
\{l, l+1, \ldots, l+k\} \cap\{i \in \mathbb{N}:\{i, i+1, \ldots, i+n\} \subseteq A\} \neq \emptyset .
$$

De (2), se tiene que:

$$
\{k, k+1, \ldots\} \subseteq\{i \in \mathbb{N}:\{i, i+1, \ldots, i+n\} \subseteq A\} .
$$

Además,

$$
l+k \in\{l, l+1, \ldots, l+k\} \quad \text { y } \quad l+k \in\{k, k+1, \ldots\} .
$$

Así, $\{l, l+1, \ldots, l+k\} \cap\{k, k+1, \ldots\} \neq \emptyset$. Luego, por (3), se obtiene que:

$$
\{l, l+1, \ldots, l+k\} \cap\{i \in \mathbb{N}:\{i, i+1, \ldots, i+n\} \subseteq A\} \neq \emptyset .
$$

Por lo tanto, $A$ es thickly sindético.

\section{Proposición 6}

Sean $A$ y $B$ subconjuntos de $\mathbb{Z}_{+}$. Si $A$ es sindético y $B$ es thick, entonces $A \cap B \neq \emptyset$.

Demostración. Supongamos que $A$ es sindético y $B$ es thick. Como $A$ es sindético, existe $l_{0} \in \mathbb{N}$ tal que, para todo $m \in \mathbb{N}$,

$$
\left\{m, m+1, \ldots, m+l_{0}\right\} \cap A \neq \emptyset .
$$

Por otro lado, como $B$ es thick y $l_{0} \in \mathbb{N}$, existe $m_{0} \in \mathbb{N}$ tal que:

$$
\left\{m_{0}, m_{0}+1, \ldots, m_{0}+l_{0}\right\} \subseteq B .
$$

De (4), aplicado a $m_{0}$ se cumple que:

$$
\left\{m_{0}, m_{0}+1, \ldots, m_{0}+l_{0}\right\} \cap A \neq \emptyset .
$$

Así, de (5), se obtiene que:

$$
\left\{m_{0}, m_{0}+1, \ldots, m_{0}+l_{0}\right\} \cap A \subseteq B \cap A .
$$

Por lo tanto, de (6) se concluye que $B \cap A \neq \emptyset$.

\section{Ejemplo 6}

Por la Proposición 4 se obtiene que 1, 2 y 3 del Ejemplo 5, son conjuntos thick y conjuntos thickly sindético.

Una propiedad que cumplen los conjuntos que son thickly sindéticos es la siguiente.

\section{Proposición 7}

Sean $A$ y $B$ subconjuntos de $\mathbb{Z}_{+}$. Si $A$ y $B$ son thickly sindéticos, entonces $A \cap B$ es thickly sindético. 
Demostración. Supongamos que $A$ y $B$ son thickly sindéticos. Probemos que $A \cap B$ es thickly sindético. Sea $n \in \mathbb{N}$. Veamos que el conjunto

$$
\{i \in \mathbb{N}:\{i, i+1, \ldots, i+n\} \subseteq A \cap B\}
$$

es sindético. Como $A$ es thickly sindético, se tiene que el conjunto $\{i \in \mathbb{N}:\{i, i+1, \ldots, i+n\} \subseteq A\}$ es sindético. Luego, existe $m_{1} \in \mathbb{N}$, tal que para todo $l \in \mathbb{N}$, se satisface que:

$$
\left\{l, l+1, \ldots, l+m_{1}\right\} \cap\{i \in \mathbb{N}:\{i, i+1, \ldots, i+n\} \subseteq A\} \neq \emptyset .
$$

De manera similar, como $B$ es thickly sindético, para $n+m_{1}$ se tiene que el conjunto $\{i \in \mathbb{N}:\{i, i+$ $\left.\left.1, \ldots, i+n+m_{1}\right\} \subseteq B\right\}$ es sindético. Por lo tanto, existe $m_{2} \in \mathbb{N}$, tal que para todo $l^{\prime} \in \mathbb{N}$, se tiene que:

$$
\left\{l^{\prime}, l^{\prime}+1, \ldots, l^{\prime}+m_{2}\right\} \cap\left\{i \in \mathbb{N}:\left\{i, i+1, \ldots, i+n+m_{1}\right\} \subseteq B\right\} \neq \emptyset .
$$

Definamos $m=m_{1}+m_{2}$. Sea $k \in \mathbb{N}$. Veamos que:

$$
\{k, k+1, \ldots, k+m\} \cap\{i \in \mathbb{N}:\{i, i+1, \ldots, i+n\} \subseteq A \cap B\} \neq \emptyset .
$$

De (8) aplicado a $k$, se obtiene que:

$$
\left\{k, k+1, \ldots, k+m_{2}\right\} \cap\left\{i \in \mathbb{N}:\left\{i, i+1, \ldots, i+n+m_{1}\right\} \subseteq B\right\} \neq \emptyset .
$$

Es decir, existe $k+j$ con $j \in\left\{0,1, \ldots, m_{2}\right\}$ tal que $k+j \in\left\{i \in \mathbb{N}:\left\{i, i+1, \ldots, i+n+m_{1}\right\} \subseteq B\right\}$. Luego,

$$
\left\{k+j, k+j+1, \ldots, k+j+n+m_{1}\right\} \subseteq B .
$$

Por otro lado, de (7) aplicado a $k+j$, se obtiene que

$$
\left\{k+j, k+j+1, \ldots, k+j+m_{1}\right\} \cap\{i \in \mathbb{N}:\{i, i+1, \ldots, i+n\} \subseteq A\} \neq \emptyset .
$$

Es decir, existe $k+j+s$, con $s \in\left\{0,1, \ldots, m_{1}\right\}$ tal que $k+j+s \in\{i \in \mathbb{N}:\{i, i+1, \ldots, i+n\} \subseteq A\}$. Luego,

$$
\{k+j+s, k+j+s+1, \ldots, k+j+s+n\} \subseteq A .
$$

Ahora, como $s \in\left\{0,1, \ldots, m_{1}\right\}$, obtenemos que:

$$
\{k+j+s, k+j+s+1, \ldots, k+j+s+n\} \subseteq\left\{k+j, k+j+1, \ldots, k+j+n+m_{1}\right\} .
$$

De donde, por (9), se tiene que:

$$
\{k+j+s, k+j+s+1, \ldots, k+j+s+n\} \subseteq B .
$$

Así, de (10), se sigue que, $\{k+j+s, k+j+s+1, \ldots, k+j+s+n\} \subseteq A \cap B$. Esto implica que $k+j+s \in\{i \in \mathbb{N}:\{i, i+1, \ldots, i+n\} \subseteq A \cap B\}$. Además, $k+j+s \in\{k, k+1, \ldots, k+m\}$. Luego,

$$
\{k, k+1, \ldots, k+m\} \cap\left\{i \in \mathbb{N}:\left\{i, i+1, \ldots, i+n+m_{1}\right\} \subseteq A \cap B\right\} \neq \emptyset .
$$

Por lo tanto, $A \cap B$ es thickly sindético.

Como consecuencia de la Proposición 7, se obtiene el Corolario 1. 
Corolario 1 Si para cada $i \in\{1,2, \ldots, k\}, A_{i} \subseteq \mathbb{Z}_{+}$es thickly sindético, entonces $\bigcap_{i=1}^{k} A_{i}$ es thickly sindético.

A continuación presentamos más clases de sistemas dińamicos discretos.

\section{Definición 7}

Sea $(X, f)$ un sistema dinámico discreto. Se dice que el sistema dinámico discreto $(X, f)$ es:

1. Sensitivo o sensible a las condiciones iniciales si existe $\delta>0$ tal que para todo $x \in X$ y para todo abierto $U$ en $X$ con $x \in U$, existen $y \in U$ y $k \in \mathbb{N}$ tales que $d\left(f^{k}(x), f^{k}(y)\right)>\delta$. A la constante $\delta$ se le llama constante de sensibilidad de $f$.

2. Li-Yorke sensitivo si existe $\delta>0$ tal que para todo $x \in X$ y para todo abierto $U$ en $X$ con $x \in U$, existe $y \in U$ tal que:

$$
\liminf _{n \rightarrow \infty} d\left(f^{n}(x), f^{n}(y)\right)=0 \quad y \quad \limsup _{n \rightarrow \infty} d\left(f^{n}(x), f^{n}(y)\right) \geq \delta .
$$

3. Multi-sensitivo si existe $\delta>0$ tal que para cualesquiera abiertos $U_{1}, U_{2}, \ldots, U_{k}$ no vacíos en $X$ se tiene que $\bigcap_{i=1}^{k} N_{f}\left(U_{i}, \delta\right) \neq \emptyset$.

4. Cofinitamente sensitivo si existe $\delta>0$ tal que para cada abierto no vacío $U$ en $X$, se tiene que $N_{f}(U, \delta)$ es cofinito.

5. Thick sensitivo si existe $\delta>0$ tal que para cada abierto no vacío $U$ en $X$, se tiene que $N_{f}(U, \delta)$ es thick.

6. Sindéticamente sensitivo si existe $\delta>0$ tal que para cada abierto no vacío $U$ en $X$, se tiene que $N_{f}(U, \delta)$ es sindético.

7. Thickly sindéticamente sensitivo (TSS) si existe $\delta>0$ tal que para cada abierto no vacío $U$ en $X$, se tiene que $N_{f}(U, \delta)$ es thickly sindético.

\section{Observación 3}

Con respecto a la definición 1, dada en la definición 7 se tiene que en términos prácticos, que $f$ sea una función sensitiva implica que si se usan sus iteraciones para modelar el comportamiento a largo plazo, entonces cualquier variación de las condiciones iniciales puede dar lugar a grandes diferencias entre el comportamiento previsto. La sensibilidad de una función, es un ingrediente clave del caos para sistemas dinámicos (vea [6]).

A continuación presentaremos un ejemplo de un sistema dinámico sensible. Posteriormente, daremos un ejemplo de los demás tipos de sistemas.

\section{Ejemplo 7}

Consideremos el sistema dinámico $(X, f)$, donde $X=[0,1]$ es el intervalo cerrado considerado con la métrica del subespacio de la recta real y la función $f: X \rightarrow X$ dada por $f(x)=2 x$, para cada $x \in X$. Se tiene que el sistema $(X, f)$ es sensitivo. En efecto, sean $\epsilon>0, x, y \in[0,1]$ tal que $|x-y|=\epsilon$. Dado que la sucesión $\left\{2^{n}\right\}_{n \in \mathbb{N}}$ no es convergente (vea [2, pág. 431]) existe $N_{\epsilon} \in \mathbb{N}$ 
tal que, para cada $n \geq N_{\epsilon}$ se sigue que

$$
2^{n}>N_{\epsilon} \text {. }
$$

Sea $n_{0} \geq N_{\epsilon}$. Así, por definición de la función se obtiene que

$$
\left|f^{n_{0}}(x)-f^{n_{0}}(y)\right|=2^{n_{0}}|x-y|=2^{n_{0}} \cdot \epsilon .
$$

En consecuencia, por (11) se sigue que

$$
\left|f^{n_{0}}(x)-f^{n_{0}}(y)\right|>N_{\epsilon} \cdot \epsilon \geq N_{\epsilon} .
$$

Finalmente, de (13) tenemos que $\left|f^{n_{0}}(x)-f^{n_{0}}(y)\right|>N_{\epsilon}$. Así, considerando $\delta=N_{\epsilon}$ se obtiene lo deseado.

\section{Ejemplo 8}

Sea $X$ un espacio métrico, compacto y conexo. Consideremos $i d_{X}: X \rightarrow X$ la función identidad. Se tiene que $\left(X, i d_{X}\right)$ no es sensitivo.

La Proposición 8, muestra una equivalencia de sistema dinámico sensitivo, la cual nos será útil en la prueba de la Proposición 10.

\section{Proposición 8}

Sea $(X, f)$ un sistema dinámico discreto. Las condiciones siguientes son equivalentes:

1. $(X, f)$ es sensitivo.

2. Existe $\delta>0$, tal que para todo subconjunto abierto no vacío $U$ de $X$, el conjunto $N_{f}(U, \delta) \neq$ $\emptyset$.

Demostración. Veamos que (1) implica (2). Supongamos que $(X, f)$ es sensitivo, con constante de sensitividad $\delta_{1}$. Sea $\delta=\delta_{1}$. Veamos que para todo subconjunto abierto no vacío $U$ de $X$, el conjunto $N_{f}(U, \delta) \neq \emptyset$. Para esto, sea $U$ un subconjunto abierto no vacío de $X$. Como $U$ es no vacío, existe $x \in X$ tal que $x \in U$. Por hipótesis, existen $y \in U$ y $n \in \mathbb{N}$ tales que $d\left(f^{n}(x), f^{n}(y)\right)>\delta$. Así, $n \in N_{f}(U, \delta)$. Por lo tanto, $N_{f}(U, \delta) \neq \emptyset$. La prueba de (2) implica (1), la haremos por contrarecíproco. Es decir, supongamos que no se cumple (1) y probaremos que no se cumple (2). Para esto, sean (1') y (2') como sigue:

$\left(1^{\prime}\right)$. Para todo $\delta>0$, existen $x \in X$ y un subconjunto abierto no vacío $U$ de $X$ con $x \in U$, tales que para todo $y \in U$ y para todo $n \in \mathbb{N}$, se cumple que $d\left(f^{n}(x), f^{n}(y)\right) \leq \delta$.

$\left(2^{\prime}\right)$. Para todo $\delta>0$, existe un subconjunto abierto no vacío $U$ de $X$ tal que el conjunto $N_{f}(U, \delta)=\emptyset$, es decir, para todo $x, y \in U$ y para todo $n \in \mathbb{N}$, se tiene que $d\left(f^{n}(x), f^{n}(y)\right) \leq \delta$.

Así, sólo basta verificar que (1') implica ( $\left.2^{\prime}\right)$. Supongamos que se cumple $\left(1^{\prime}\right)$ y veamos que se cumple $\left(2^{\prime}\right)$. Para eso, sea $\delta>0$. Por hipótesis, para $\frac{\delta}{2}$, existen $x \in X$ y un subconjunto abierto no vacío $U$ de $X$ con $x \in U$, tal que para todo $y \in U$ y para todo $n \in \mathbb{N}$ se cumple que:

$$
d\left(f^{n}(x), f^{n}(y)\right) \leq \frac{\delta}{2} .
$$

Sean $z, w \in U$ y $k \in \mathbb{N}$. Aplicando (14), a los puntos $z, w$ resulta que:

$$
d\left(f^{k}(x), f^{k}(z)\right) \leq \frac{\delta}{2} \quad \text { y } \quad d\left(f^{k}(x), f^{k}(w)\right) \leq \frac{\delta}{2} .
$$


Luego,

$$
d\left(f^{k}(z), f^{k}(w)\right) \leq d\left(f^{k}(z), f^{k}(x)\right)+d\left(f^{k}(x), f^{k}(w)\right) .
$$

Así, por (15), se satisface que:

$$
d\left(f^{k}(z), f^{k}(w)\right) \leq \frac{\delta}{2}+\frac{\delta}{2}=\delta .
$$

Con todo, se cumple (2'). Por lo tanto, (2) implica (1).

\section{Relaciones entre los tipos de sensibilidad}

En esta sección mostramos las relaciones que se tienen entre los sistemas dinámicos de tipo sensitivo. Así como también se dan condiciones para que algunos de estos tipos de sistemas sean equivalentes. Lo cual nos ayuda a verificar las relaciones entre estos y los sistemas de tipo compacto transitivo.

\section{Proposición 9}

Sea $(X, f)$ un sistema dinámico discreto. Si $(X, f)$ es cofinitamente sensitivo, entonces $(X, f)$ es thickly sindéticamente sensitivo.

Demostración. Supongamos que $(X, f)$ es cofinitamente sensitivo. Veamos que $(X, f)$ es thickly sindéticamente sensitivo. Para esto, sea $U$ un subconjunto abierto no vacío en $X$. Por hipótesis, existe $\delta>0$ tal que $N_{f}(U, \delta)$ es cofinito. Luego, por la Proposición 5, se tiene que $N_{f}(U, \delta)$ es thickly sindético. Por lo tanto, $(X, f)$ es thickly sindéticamente sensitivo.

\section{Proposición 10}

Sea $(X, f)$ un sistema dinámico discreto. $\mathrm{Si}(X, f)$ es thick sensitivo, entonces $(X, f)$ es sensitivo.

Demostración. Supongamos que $(X, f)$ es thick sensitivo con constante de sensitividad $\delta>0$. Sea $U$ un subconjunto abierto no vacío en $X$. Por hipótesis $N_{f}(U, \delta)$ es thick. Luego, $N_{f}(U, \delta) \neq \emptyset$. Finalmente, por la Proposición 8 , se concluye que $(X, f)$ es sensitivo.

Para probar el Teorema 2 antes mencionaremos el siguiente Teorema 1 el cual fue tomado de [7] y adaptado para nuestros fines.

\section{Teorema 1}

Sea $(X, f)$ un sistema dinámico discreto. Las condiciones siguientes son equivalentes:

1. $(X, f)$ es débilmente mezclante.

2. Para cada $U_{1}, U_{2}, V_{1}$ y $V_{2}$ abiertos no vacíos en $X$, existen subconjuntos abiertos no vacíos $U$ y $V$, en $X$ tales que:

$$
N_{f}(U, V) \subseteq N_{f}\left(U_{1}, V_{1}\right) \cap N_{f}\left(U_{2}, V_{2}\right) \text { y } N_{f}(U, V) \neq \emptyset .
$$


3. Para cada $k \in \mathbb{N}$ y para cualesquiera abiertos no vacíos $U_{1}, \ldots, U_{k}$ y $V_{1}, \ldots, V_{k}$ de $X$, existe $n \in \mathbb{Z}_{+}$tal que $n \in \bigcap_{i=1}^{k} N_{f}\left(U_{i}, V_{i}\right)$.

4. Para cada par de subconjuntos abiertos no vacíos $U$ y $V$ de $X$, se cumple que el conjunto $N_{f}(U, V)$ es thick.

5. Para cada par de subconjuntos abiertos no vacíos $U$ y $V$ de $X$, existe $n \in \mathbb{N}$ tal que:

$$
U \cap f^{-n}(U) \neq \emptyset \mathrm{y} U \cap f^{-n}(V) \neq \emptyset .
$$

\section{Teorema 2}

Sea $(X, f)$ un sistema dinámico discreto. Si $(X, f)$ es débilmente mezclante, entonces $(X, f)$ es thick sensitivo.

Demostración. Supongamos que $(X, f)$ es débilmente mezclante. Probemos que $(X, f)$ es thick sensitivo. Sean $\delta \in(0, \operatorname{diám}(X) / 4)$ y $W$ un abierto en $X$. Veamos que $N_{f}(W, \delta)$ es thick. Como $\delta>0$, existen $x_{0}, y_{0} \in X$ tales que:

$$
\operatorname{diám}(X)-\delta<d\left(x_{0}, y_{0}\right) .
$$

Dado que $\delta<\frac{\operatorname{diám}(\mathrm{X})}{4}$, se tiene que $4 \delta-\delta<\operatorname{diám}(X)-\delta<d\left(x_{0}, y_{0}\right)$. Luego,

$$
3 \delta<d\left(x_{0}, y_{0}\right) .
$$

Sean $U=B\left(x_{0}, \delta\right)$ y $V=B\left(y_{0}, \delta\right)$. Sean $x \in U$ y $y \in V$. Por la desigualdad del triángulo se tiene que:

$$
d\left(x_{0}, y_{0}\right) \leq d\left(x_{0}, x\right)+d(x, y)+d\left(y, y_{0}\right) .
$$

Así, por (16), resulta que:

$$
3 \delta<d\left(x_{0}, y_{0}\right) \leq \delta+d(x, y)+\delta .
$$

Esto implica que $\delta<d(x, y)$. Dado que $x$ y y son puntos arbitrarios de $U$ y $V$, respectivamente, se concluye que para todo $x \in U$ y para todo $y \in V$,

$$
\delta<d(x, y)
$$

Ahora, puesto que $(X, f)$ es débilmente mezclante por el inciso (2) del Teorema 1, para los conjuntos abiertos $U, V$ y $W$, existen abiertos $U^{\prime}$ y $V^{\prime}$ no vacíos en $X$ tales que:

$$
N_{f}\left(U^{\prime}, V^{\prime}\right) \neq \emptyset \mathrm{y} N_{f}\left(U^{\prime}, V^{\prime}\right) \subseteq N_{f}(W, U) \cap N_{f}(W, V) .
$$

Probemos ahora que $N_{f}(W, U) \cap N_{f}(W, V) \subseteq N_{f}(W, \delta)$. Para esto sea $n \in N_{f}(W, U) \cap N_{f}(W, V)$. Demostraremos que

$$
n \in N_{f}(W, \delta) .
$$

Es decir, veamos que:

$$
n \in \mathbb{Z}_{+} \text {y existen } x, y \in U \text { tales que } d\left(f^{n}(x), f^{n}(y)\right)>\delta .
$$

Como $n \in N_{f}(W, U) \cap N_{f}(W, V)$ se tiene que $W \cap f^{-n}(U) \neq \emptyset \mathrm{y} W \cap f^{-n}(V) \neq \emptyset$. Luego, de la Proposición 1, obtenemos que:

$$
f^{n}(W) \cap U \neq \emptyset \text { y } f^{n}(W) \cap V \neq \emptyset .
$$


Por lo tanto, existen $x, y \in W$ tales que:

$$
f^{n}(x) \in U \text { y } f^{n}(y) \in V
$$

De (17), obtenemos que $d\left(f^{n}(x), f^{n}(y)\right)>\delta$. En consecuencia (19) queda probado. Por lo tanto,

$$
N_{f}(W, U) \cap N_{f}(W, V) \subseteq N_{f}(W, \delta) .
$$

Finalmente, por (18) y (20) se satisface que:

$$
N_{f}\left(U^{\prime}, V^{\prime}\right) \subseteq N_{f}(W, \delta)
$$

Como $(X, f)$ es débilmente mezclante aplicando nuevamente el inciso (4) del Teorema 1, a los conjuntos abiertos $U^{\prime}$ y $V^{\prime}$ resulta que $N_{f}\left(U^{\prime}, V^{\prime}\right)$ es thick. Luego, por la Proposición 3, se concluye que $N_{f}(W, \delta)$ es thick.

De la Proposición 10 y del Teorema 2, se obtiene el siguiente resultado.

Corolario 2 Sea $(X, f)$ un sistema dinámico discreto. Si $(X, f)$ es débilmente mezclante, entonces $(X, f)$ es sensitivo.

El Teorema 3 nos muestra la relación que existe entre la clase de los sistemas thickly sindéticamente sensitivo y los multi-sentitivos.

\section{Teorema 3}

Sea $(X, f)$ un sistema dinámico discreto. Si $(X, f)$ es thickly sindéticamente sensitivo, entonces $(X, f)$ es multi-sensitivo.

Demostración. Supongamos que $(X, f)$ es thickly sindéticamente sensitivo con constante de sensitividad $\delta>0$. Veamos que $(X, f)$ es multi-sensitivo. Sea $k \in \mathbb{N}$. Para $i \in\{1,2 \ldots, k\}$, sea $U_{i}$ un subconjunto abierto no vacío en $X$. Veamos que $\bigcap_{i=1}^{k} N_{f}\left(U_{i}, \delta\right) \neq \emptyset$. Por hipótesis, para cada $i \in\{1, \ldots, k\}$, se tiene que $N_{f}\left(U_{i}, \delta\right)$ es thickly sindético. En consecuencia, por el Corolario 1 , se deduce que $\bigcap_{i=1}^{k} N_{f}\left(U_{i}, \delta\right)$ es thickly sindético. Así, se concluye que:

$$
\bigcap_{i=1}^{k} N_{f}\left(U_{i}, \delta\right) \neq \emptyset
$$

Por lo tanto, $(X, f)$ es multi-sensitivo.

\section{Teorema 4}

Sea $(X, f)$ un sistema dinámico discreto. Si $(X, f)$ es multi-sensitivo, entonces $(X, f)$ es thick sensitivo. 
Demostración. Supongamos que $(X, f)$ es multi-sensitivo con constante de sensitividad $\delta$. Verifiquemos que $(X, f)$ es thick sensitivo. Sea $U$ un subconjunto abierto y no vacío en $X$. Veamos que $N_{f}(U, \delta)$ es thick. Sea $k \in \mathbb{N}$. Veamos que existe $n \in \mathbb{N}$ tal que $\{n, n+1, \ldots, n+k\} \subseteq N_{f}(U, \delta)$. Para cada $i \in\{0,1, \ldots, k\}$ sea

$$
U_{i} \subseteq f^{-i}(U) \text { tal que diám }\left(f^{j}\left(U_{i}\right)\right)<\delta \text {, para cada } j \in\{0,1, \ldots, k\} .
$$

Como $(X, f)$ es multi-sensitivo, se tiene que $\bigcap_{i=0}^{k} N_{f}\left(U_{i}, \delta\right) \neq \emptyset$. Luego, existe $l \in \mathbb{N}$ tal que $l \in \bigcap_{i=0}^{k} N_{f}\left(U_{i}, \delta\right)$. Así,

$$
l \in N_{f}\left(U_{i}, \delta\right) \text {, para todo } i \in\{0,1, \ldots, k\} .
$$

Luego, para cada $i \in\{0,1, \ldots, k\}$, existen $x_{i}, y_{i} \in U_{i}$ tales que $d\left(f^{l}\left(x_{i}\right), f^{l}\left(y_{i}\right)\right)>\delta$. Por el inciso (3) de la Observación 2 y de (21), se obtiene que:

$$
l \in \bigcap_{i=0}^{k} N_{f}\left(f^{-i}(U), \delta\right)
$$

Nuevamente de (21), se deduce que $l>k$. Luego, $l-i+i \in N_{f}\left(f^{-i}(U), \delta\right)$. Por la Proposición 2 y (22), resulta que $l-i \in N_{f}\left(U_{i}, \delta\right)$, para todo $i \in\{0,1, \ldots, k\}$. Luego,

$$
\{l, l-1, \ldots, l-k\} \subseteq N_{f}(U, \delta) .
$$

Así, haciendo $n=l-k>0$, se concluye que $\{n, n+1, \ldots, n+k\} \subseteq N_{f}(U, \delta)$. Por lo tanto, $N_{f}(U, \delta)$ es thick.

Del la Proposición 10 y del Teorema 4, se obtiene el siguiente resultado.

Corolario 3 Sea $(X, f)$ un sistema dinámico discreto. Si $(X, f)$ es multi-sensitivo, entonces $(X, f)$ es sensitivo.

Una prueba alternativa del Corolario 3, que puede servir al lector, es la siguiente.

\section{Teorema 5}

Sea $(X, f)$ un sistema dinámico discreto. $\mathrm{Si}(X, f)$ es multi-sensitivo, entonces $(X, f)$ es sensitivo.

Demostración. Supongamos que $(X, f)$ es multi-sensitivo, con constante de sensitividad $\delta>0$. Demostremos que $(X, f)$ es sensitivo aplicando la Proposición 8 . Sea $U$ un subconjunto abierto y no vacío en $X$. Sean $k \in \mathbb{N}$ y $U_{i}=U$, para todo $i \in\{1,2, \ldots, k\}$. Por hipótesis

$$
\bigcap_{i=1}^{k} N_{f}\left(U_{i}, \delta\right) \neq \emptyset
$$

Esto implica que, $N_{f}(U, \delta) \neq \emptyset$. Así, se tiene que $(X, f)$ es sensitivo.

El Teorema 6 nos indica que si el espacio $X$ coincide con el intervalo $[0,1]$, entonces el sistema dinámico sensitivo es cofinitamente sensitivo. Una prueba de dicho resultado la puede consultar en [17, Teorema 2]. 


\section{Teorema 6}

Sea $([0,1], f)$ un sistema dinámico discreto. Si $([0,1], f)$ es sensitivo, entonces $([0,1], f)$ es cofinitamente sensitivo.

Como consecuencia del Teorema 6 y de la Figura 7, se obtiene el corolario siguiente.

Corolario 4 Sea $([0,1], f)$ un sistema dinámico discreto. Los enunciados siguientes son equivalentes:

1. $([0,1], f)$ es cofinitamente sensitivo.

2. $([0,1], f)$ es thickly sindéticamente sensitivo.

3. $([0,1], f)$ es multi-sensitivo.

4. $([0,1], f)$ es thick sensitivo.

5. $([0,1], f)$ es sensitivo.

En la Figura 7 se muestran relaciones que se cumplen entre los sistemas dinámicos de tipo sensitivo. La flecha significa que la clase de funciones precedente implica la siguiente.

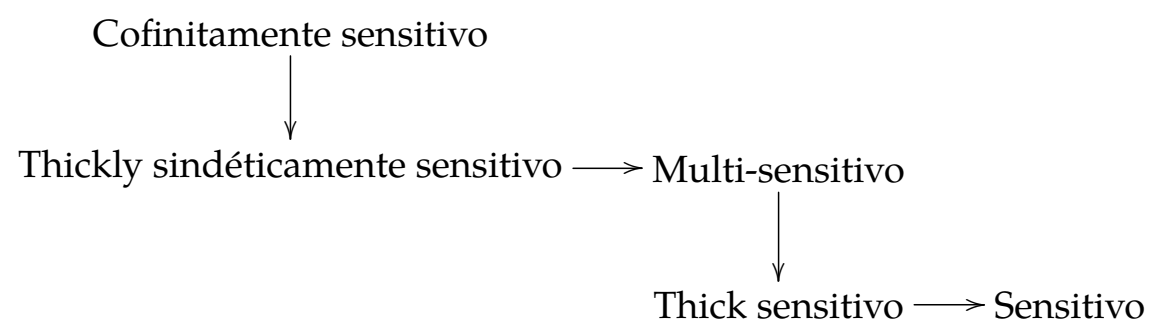

Figura 7: Diagrama que muestra las relaciones entre los sistemas dinámicos del tipo sensitivo.

El Teorema 7 nos dice cuándo algunas de las relaciones de la Figura 7 resultan ser equivalentes. Antes recordemos que $X$ es un $M$-sistema si el sistema es transitivo y el conjunto de los puntos casiperiódicos es denso en $X$.

Teorema 7

Sea $(X, f)$ un sistema dinámico. Si $(X, f)$ es un M-sistema, entonces los enunciados siguientes son equivalentes:

1. $(X, f)$ es multi-sensitivo.

2. $(X, f)$ es thickly sindéticamente sensitivo.

3. $(X, f)$ es thick sensitivo.

Del Ejemplo 8 se sabe que el sistema dinámico $\left(X, i d_{X}\right)$ no es sensitivo. En consecuencia de la Figura 7 , se obtiene el siguiente ejemplo.

\section{Ejemplo 9}

Consideremos el sistema dinámico $\left(X, i d_{X}\right)$, donde $i d_{X}: X \rightarrow X$ es la función identidad. Por la Figura 7, se tiene que:

1. $\left(X, i d_{X}\right)$ no es cofinitamente sensitivo. 
2. $\left(X, i d_{X}\right)$ no es thickly sindéticamente sensitivo.

3. $\left(X, i d_{X}\right)$ no es multi-sensitivo.

4. $\left(X, i d_{X}\right)$ no es thick sensitivo.

A continuación, mencionamos un resultado que nos permite relacionar los sistemas dinámicos mezclantes con los sistemas de tipo sensitivo. Este resultado lo puede verificar en [17, Proposición 2].

\section{Proposición 11}

Sea $(X, f)$ un sistema dinámico. $\operatorname{Si}(X, f)$ es mezclante, entonces para cualquier $\delta \in(0, \operatorname{diám}(X))$ se cumple que $(X, f)$ es cofinitamente sensitivo, con constante de sensitividad $\delta$.

De la Figura 7 y de la Proposición 11, se obtiene la Figura 8. La flecha significa que la clase de funciones precedente implica la siguiente.

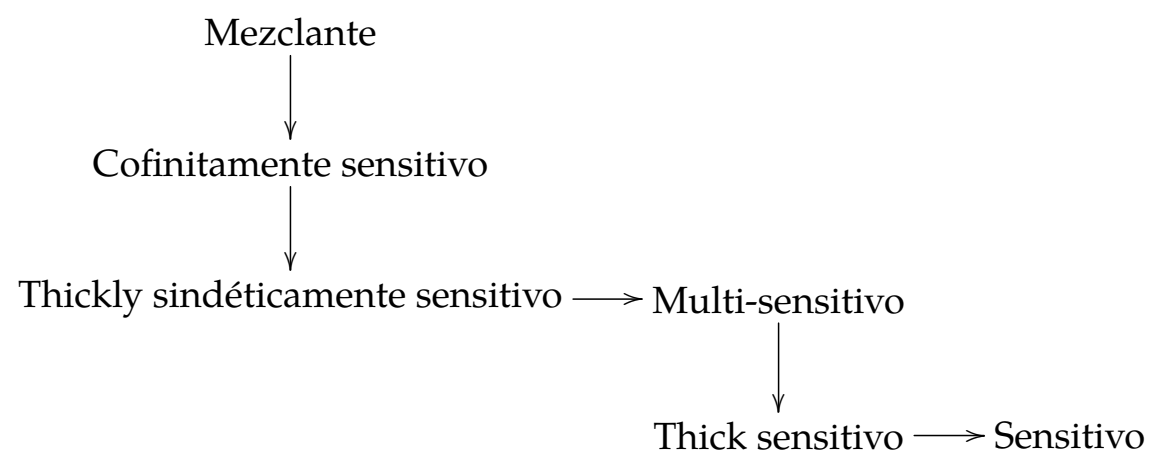

Figura 8: Diagrama que muestra las relaciones entre los sistemas dinámicos mezclantes y del tipo sensitivo.

La prueba de la Proposición 12 la puede consultar en [1, Proposición 2.1]. Dicho resultado nos ayuda a verificar otras relaciones entre estos tipos de sistemas. Antes de plantear este resultado, recordemos que el símbolo $\mathcal{O}(x, f)$ denota la órbita del punto $x$ bajo la función $f$ (vea Definición 2 ).

\section{Proposición 12}

Sea $(X, f)$ un sistema dinámico. Las condiciones siguientes son equivalentes:

1. $(X, f)$ es transitivo.

2. Existe $x \in X$ tal que $\mathcal{O}(x, f)$ es densa en $X$.

Finalizamos esta sección, con el Teorema 8, el cual establece que todo sistema dinámico thick sensitivo y transitivo es multi-sensitivo.

\section{Teorema 8}

Sea $(X, f)$ un sistema dinámico. $\operatorname{Si}(X, f)$ es thick sensitivo y transitivo, entonces $(X, f)$ es multisensitivo.

Demostración. Supongamos que $(X, f)$ es transitivo y thick sensitivo con constante de sensitividad $\delta>0$. Probemos que $(X, f)$ es multi-sensitivo. Sean $U_{1}, U_{2}, \ldots, U_{k}$ subconjuntos abiertos no vacíos de $X$. Veamos que $\bigcap_{i=1}^{k} N_{f}\left(U_{i}, \delta\right) \neq \emptyset$. Como $(X, f)$ es transitivo, por la Proposición 12 , existe $x \in X$, tal 
que $\mathcal{O}(x, f)$ es densa en $X$. Luego,

$$
\mathcal{O}(x, f) \cap U_{i} \neq \emptyset \text {, para todo } i \in\{1,2, \ldots, k\} .
$$

Así, para cada $i \in\{1,2, \ldots, k\}$, existe $n_{i} \in \mathbb{N}$ tal que:

$$
f^{n_{i}}(x) \in U_{i} \text {, para cada } i \in\{1,2, \ldots, k\} .
$$

Definamos $U=\bigcap_{i=1}^{k} f^{-n_{i}}\left(U_{i}\right)$. Notemos que de (23), se deduce que $x \in f^{-n_{i}}\left(U_{i}\right)$, para cada $i \in$ $\{1,2, \ldots, k\}$. Luego, $x \in \bigcap_{i=1}^{k} f^{-n_{i}}\left(U_{i}\right)=U$. Así, $U$ es no vacío. Además, por la continuidad de $f$, resulta que $f^{n_{i}}$ es continua, para todo $i \in\{1,2, \ldots, k\}$. Así, $f^{-n_{i}}\left(U_{i}\right)$, para cada $\{i \in\{1,2, \ldots, k\}$, es un conjunto abierto en $X$. Luego, $\bigcap_{i=1}^{k} f^{-n_{i}}\left(U_{i}\right)=U$ es un subconjunto abierto en $X$.

Por otro lado, notemos que para todo $i \in\{1,2, \ldots, k\}$,

$$
f^{n_{i}}(U)=f^{n_{i}}\left(\bigcap_{j=1}^{k} f^{-n_{j}}\left(U_{j}\right)\right) \subset f^{n_{i}}\left(f^{-n_{i}}\left(U_{i}\right)\right) \subset U_{i}
$$

En consecuencia, $f^{n_{i}}(U) \subset U_{i}$, para todo $i \in\{1,2, \ldots, k\}$. Esto implica que $U \subset f^{-n_{i}}\left(U_{i}\right)$. Luego, por el inciso (1) de la Observación 2, obtenemos que para todo $i \in\{1,2, \ldots, k\}$,

$$
N_{f}(U, \delta) \subset N_{f}\left(f^{-n_{i}}\left(U_{i}\right), \delta\right) .
$$

Por otro lado, como $(X, f)$ es thick sensitivo, se satisface que $N_{f}(U, \delta)$ es thick. Luego, para $n_{1}+\cdots+$ $n_{k} \in \mathbb{N}$, existe $s \in \mathbb{N}$ tal que:

$$
\left\{s, s+1, \ldots, s+n_{1}+\cdots+n_{k}\right\} \subset N_{f}(U, \delta) .
$$

Así, de (24), se deduce que:

$$
\left\{s, s+1, \ldots, s+n_{1}+\cdots+n_{k}\right\} \subset N_{f}\left(f^{-n_{i}}\left(U_{i}\right), \delta\right) .
$$

De donde, $s+n_{i} \in N_{f}\left(f^{-n_{i}}\left(U_{i}\right), \delta\right)$, para todo $i \in\{1,2, \ldots, k\}$. Luego, por la Proposición 2, se concluye que $s \in N_{f}\left(U_{i}, \delta\right)$, para todo $i \in\{1,2, \ldots, k\}$. En consecuencia, $s \in \bigcap_{i=1}^{k} N_{f}\left(U_{i}, \delta\right)$. Por lo tanto, $(X, f)$ es multi-sensitivo.

\section{Compacto transitividad}

En esta sección se introduce la noción del conjunto omega-límite de un punto con respecto a una familia de Furstenberg. Dicho conjunto nos ayudará a definir los sistemas dinámicos compacto transitivo. Antes de presentar estos conceptos damos algunos conceptos que nos ayudan a definir este tipo de sistemas. Acordemos que en este apartado denotaremos por $\mathcal{P}\left(\mathbb{Z}_{+}\right)$a el conjunto de todos los subconjuntos de $\mathbb{Z}_{+}$. 


\section{Definición 8}

Un subconjunto $\mathcal{F}$ de $\mathcal{P}\left(\mathbb{Z}_{+}\right)$es una familia de Furstenberg, si para cualesquiera $F_{1}, F_{2} \in \mathcal{P}\left(\mathbb{Z}_{+}\right)$ tales que $F_{1} \subseteq F_{2}$ y $F_{1} \in \mathcal{F}$ se cumple que $F_{2} \in \mathcal{F}$.

Para ilustrar la Definición 8 veamos algunos ejemplos.

\section{Ejemplo 10}

Sea $A \in \mathcal{P}\left(\mathbb{Z}_{+}\right)$tal que $A \neq \emptyset$. La familia

$$
\mathcal{F}_{A}=\left\{F \in \mathcal{P}\left(\mathbb{Z}_{+}\right): A \subseteq F\right\},
$$

es una familia de Furstenberg. En efecto, sean $F_{1}, F_{2} \in \mathcal{P}\left(\mathbb{Z}_{+}\right)$de tal manera que $F_{1} \subset F_{2}$ y $F_{1} \in \mathcal{F}_{A}$. Luego $A \subseteq F_{1} \subseteq F_{2}$. Así, $A \subseteq F_{2}$. Por lo tanto, $F_{2} \in \mathcal{F}_{A}$.

\section{Definición 9}

Sea $(X, f)$ un sistema dinámico. Se define la familia $\mathcal{N}_{f}$ como sigue:

$$
\mathcal{N}_{f}=\left\{A \subseteq \mathbb{Z}_{+}: \text {existen } U, V \subseteq X \text { abiertos no vacíos, tales que } N_{f}(U, V) \subseteq A\right\} .
$$

\section{Observación 4}

Sea $(X, f)$ un sistema dinámico. Para cualesquiera $U$ y $V$ subconjuntos abiertos no vacíos en $X$, se tiene que $N_{f}(U, V) \in \mathcal{N}_{f}$. De donde, $\mathcal{N}_{f} \neq \emptyset$.

\section{Ejemplo 11}

La familia $\mathcal{N}_{f}$ dada en la Definición 9, es de Furstenberg.

En efecto, sean $F_{1}, F_{2} \in \mathcal{N}_{f}$ tales que $F_{1} \subseteq F_{2}$ y $F_{1} \in \mathcal{N}_{f}$. Luego, existen $U$ y $V$ subconjuntos abiertos no vacíos en $X$ tales que $N_{f}(U, V) \subseteq F_{1}$. Como $F_{1} \subseteq F_{2}, N_{f}(U, V) \subseteq F_{2}$. Por lo tanto, $F_{2} \in \mathcal{N}_{f}$.

\section{Proposición 13}

Sea $F \in \mathcal{P}\left(\mathbb{Z}_{+}\right)$. Se cumple que $F \cap R \neq \emptyset$, para todo $R \in \mathcal{N}_{f}$ si y solo si $F \cap N_{f}(U, V) \neq \emptyset$, para todos $U$ y $V$ subconjuntos abiertos no vacíos en $X$.

Demostración. Supongamos que $F \cap F^{\prime} \neq \emptyset$, para todo $F^{\prime} \in \mathcal{N}_{f}$. Sean $U$ y $V$ subconjuntos abiertos no vacío de $X$. Por la Observación 4, se tiene que $N_{f}(U, V) \in \mathcal{N}_{f}$. Así, por el supuesto, se tiene que $F \cap N_{f}(U, V) \neq \emptyset$. Recíprocamente, supongamos que $F \cap N_{f}(U, V) \neq \emptyset$, para todos $U$ y $V$ subconjuntos abiertos no vacíos de $X$. Sea $F^{\prime} \in \mathcal{N}_{f}$. Por la Definición 9, existen subconjuntos abiertos no vacíos $U$ y $V$ de $X$, tales que $N_{f}(U, V) \subseteq F^{\prime}$. Luego $N_{f}(U, V) \cap F \subseteq F^{\prime} \cap F$. Por hipótesis $N_{f}(U, V) \cap F \neq \emptyset$. Por lo tanto, $F^{\prime} \cap F \neq \emptyset$. 
Definición 10

Sea $\mathcal{F}$ una familia de Furstenberg. La familia dual de $\mathcal{F}$, denotada por $k \mathcal{F}$, se define como:

$$
k \mathcal{F}=\left\{F \in \mathcal{P}\left(\mathbb{Z}_{+}\right): F \cap R \neq \emptyset \text {, para cualquier } R \in \mathcal{F}\right\} .
$$

Ilustramos el concepto dado en la Definición 10 mediante los siguientes ejemplos, Ejemplo 12 y Ejemplo 13.

\section{Ejemplo 12}

La familia dual de la familia $\mathcal{F}_{A}$ dada en el Ejemplo 10 es:

$$
\begin{aligned}
k \mathcal{F}_{A} & =\left\{F \in \mathcal{P}\left(\mathbb{Z}_{+}\right): F \cap R \neq \emptyset, \text { para todo } R \in \mathcal{F}_{A}\right\} \\
& =\left\{F \in \mathcal{P}\left(\mathbb{Z}_{+}\right): F \cap A \neq \emptyset\right\} \\
& =\left\{F \subseteq \mathbb{Z}_{+}: \text {existe } a \in F \text { y } a \in A\right\}
\end{aligned}
$$

\section{Ejemplo 13}

La familia dual de la familia $\mathcal{N}_{f}$ dada en la Definición 9, es:

$$
k \mathcal{N}_{f}=\left\{F \in \mathcal{P}\left(\mathbb{Z}_{+}\right): F \cap R \neq \emptyset \text {, para cualquier } R \in \mathcal{N}_{f}\right\} .
$$

Por la Proposición 13, se tiene que:

$$
k \mathcal{N}_{f}=\left\{F \in \mathcal{P}\left(\mathbb{Z}_{+}\right): F \cap N_{f}(U, V) \neq \emptyset \text {, para todos } U, V \subseteq X \text {, abiertos no vacíos }\right\} .
$$

\section{Definición 11}

Sean $(X, f)$ un sistema dinámico, $\mathcal{F} \subseteq \mathcal{P}\left(\mathbb{Z}_{+}\right)$una familia de Furstenberg y $x \in X$. El conjunto $\omega$-limite de $x$ con respecto a la familia $\mathcal{F}$, denotado por $\omega_{\mathcal{F}}(x)$, se define como:

$\omega_{\mathcal{F}}(x)=\left\{z \in X: N_{f}(x, G) \in k \mathcal{F}\right.$, para cada vecindad $G$ de $\left.z\right\}$.

\section{Ejemplo 14}

Sean $(X, f)$ un sistema dinámico y $x \in X$. Por la Definición 11 y la Definición 9 , se tiene que: $\omega_{\mathcal{N}_{f}}(x)=\left\{z \in X: N_{f}(x, G) \in k \mathcal{N}_{f}\right.$, para cada vecindad $G$ de $\left.z\right\}$.

Ocupando (25) del Ejemplo 13 y el Ejemplo 14, se obtiene la siguiente observación.

\section{Observación 5}

El conjunto $\omega$-límite de $x$, respecto a la familia $\mathcal{N}_{f}$ se puede escribir de la siguiente forma:

$$
\omega_{\mathcal{N}_{f}}(x)=\left\{z \in X: N_{f}(x, G) \cap N_{f}(U, V) \neq \emptyset \text {, para cada vecindad } G \text { de } z\right.
$$

y para cualesquiera $U, V \subseteq X$ abiertos no vacíos $\}$.

En [11, Lema 3.3.11] encontrará una prueba detallada del Lema 1. 
Lema 1 Sean $(X, f)$ un sistema dinámico débilmente mezclante, $W$ un subconjunto cerrado y no vacío en $X$ y $x \in X$. Si $N_{f}(x, W)$ es sindético, entonces $\omega_{\mathcal{N}_{f}}(x) \cap W \neq \emptyset$.

Terminamos esta sección dando el concepto de sistema dinámico compacto transitivo y mostrando una equivalencia del mismo.

\section{Definición 12}

Sea $(X, f)$ un sistema dinámico. Se dice que $(X, f)$ es compacto transitivo si para cada $x \in X$, existe $z \in X$ tal que $N_{f}(x, G) \cap N_{f}(U, V) \neq \emptyset$, para cada vecindad $G$ de $z$ y para cualesquiera abiertos no vacíos $U$ y $V$ de $X$.

En la Figura 9, se muestra de manera geométrica el concepto de compacto transitividad (Definición 12). Básicamente el concepto nos dice que, dado un punto $x \in X$, existe un punto $z \in X$, tal que para cualquier vecindad de $z$, digamos $G$ como se muestra en la Figura 9, y cualesquiera dos subconjuntos abiertos no vacíos en $X$, digamos $U$ y $V$, existe un momento en el cual, al mismo tiempo que $f^{n}(U)$ intersecta al conjunto $V$, se cumple que $f^{n}(x) \in G$.

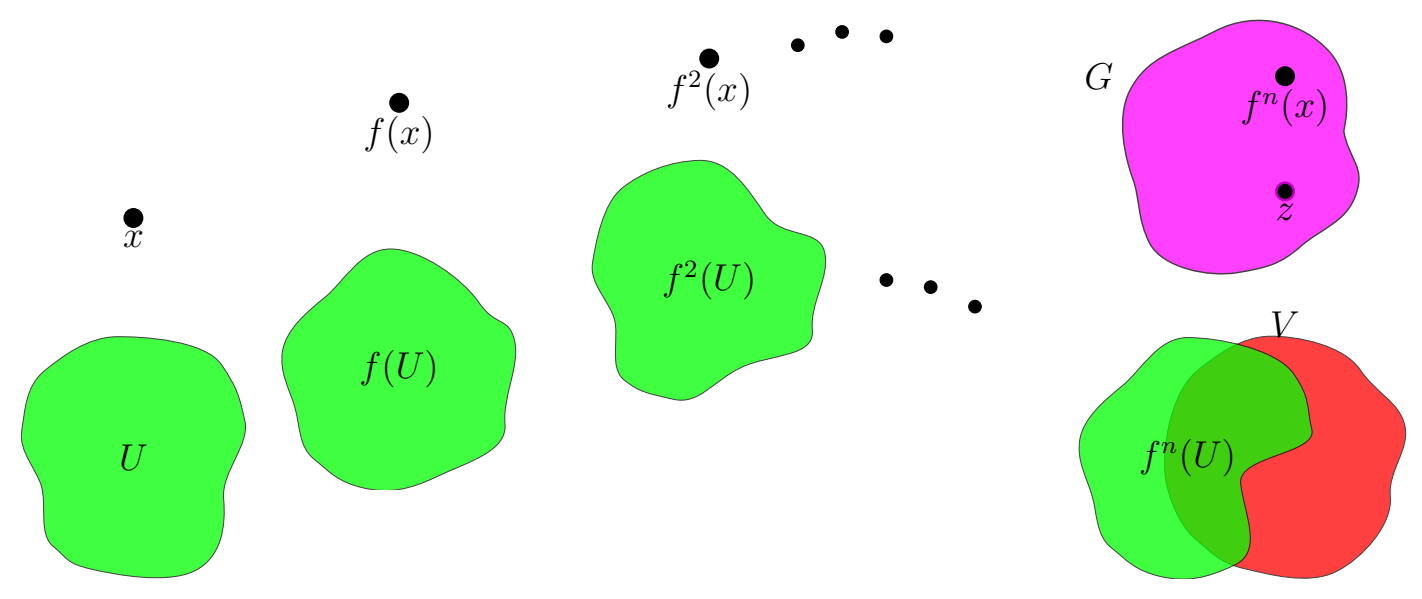

Figura 9: Representación gráfica de un sistema dinámico compacto transitivo.

De la Observación 5 y la Definición 12, se tiene el siguiente resultado.

\section{Proposición 14}

Sea $(X, f)$ un sistema dinámico. Se cumple que $(X, f)$ es compacto transitivo si y solo si $\omega_{\mathcal{N}_{f}}(x) \neq$ $\emptyset$, para cada $x \in X$.

Demostración. Supongamos que $(X, f)$ es compacto transitivo. Sea $x \in X$. Luego, existe $z \in X$ tal que $N_{f}(x, G) \cap N_{f}(U, V) \neq \emptyset$, para cada vecindad $G$ de $z$ y para cualesquiera subconjuntos abiertos no vacíos $U$ y $V$ de $X$. Así, por la Observación 5, se tiene que $z \in \omega_{\mathcal{N}_{f}}(x)$. Por lo tanto, $\omega_{\mathcal{N}_{f}}(x) \neq \emptyset$.

Recíprocamente, supongamos que $\omega_{\mathcal{N}_{f}}(x) \neq \emptyset$. Por la Observación 5, existe $z \in X$, tal que $N_{f}(x, G) \cap$ $N_{f}(U, V) \neq \emptyset$, para cada vecindad $G$ de $z$ y para cualesquiera subconjuntos abiertos no vacíos $U$ y $V$ de $X$. Por Definición 12 , se concluye que $(X, f)$ es compacto transitivo.

\section{Teorema 9}

Sea $(X, f)$ un sistema dinámico. Si $(X, f)$ es débilmente mezclante, entonces $(X, f)$ es compacto transitivo. 
Demostración. Supongamos que $(X, f)$ es débilmente mezclante. Para verificar que $(X, f)$ es compacto transitivo, por la Proposición 14, basta verificar que $\omega_{\mathcal{N}_{f}}(x) \neq \emptyset$, para cada $x \in X$. Para esto, sea $x \in X$. Notemos que $X \subseteq X$ y es cerrado en sí mismo, además $N_{f}(x, X)$ es sindético. Por el Lema 1 , se obtiene que $\omega_{\mathcal{N}_{f}}(x) \cap X \neq \emptyset$. Dado que $\omega_{\mathcal{N}_{f}}(x) \cap X=\omega_{\mathcal{N}_{f}}(x)$ tenemos que $\omega_{\mathcal{N}_{f}}(x) \neq \emptyset$. Por lo tanto, $(X, f)$ es compacto transitivo.

El Ejemplo 15 fue probado en [14, Proposición 1.6.5, pág. 44].

\section{Ejemplo 15}

La función tienda es débilmente mezclante en el intervalo [0,1].

Como consecuencia del Ejemplo 15 y del Teorema 9, se obtiene el siguiente resultado.

\section{Ejemplo 16}

La función tienda es compacto transitiva en el intervalo $[0,1]$.

\section{Compacto transitividad y su relación con tipos de sensitividad}

En esta sección estudiamos la relación entre los sistemas compacto transitivos con los sistemas del tipo sensitivo.

\section{Teorema 10}

Sea $(X, f)$ un sistema dinámico. $\mathrm{Si}(X, f)$ es compacto transitivo, entonces $(X, f)$ es sensitivo.

Demostración. Supongamos que $(X, f)$ es compacto transitivo. Demostremos que $(X, f)$ es sensitivo. Sea $0<\varepsilon<\frac{\operatorname{diám}(X)}{4}$. Esto implica que $0<4 \varepsilon<\operatorname{diám}(X)$. Luego, $0<2 \varepsilon<\frac{\operatorname{diám}(X)}{2}$. En consecuencia, $\frac{\operatorname{diám}(X)}{2}-2 \varepsilon>0$. Sea $\delta=\frac{\operatorname{diám}(X)}{2}-2 \varepsilon$. Note que $\delta>0$. Mostremos que $\delta$ es una constante de sensitividad para $(X, f)$. Sean $x \in X$ y $U_{0}$ subconjunto abierto en $X$, tal que $x \in U_{0}$. Como $(X, f)$ es compacto transitivo, existe $y \in X$ tal que:

$$
N_{f}(x, G) \cap N_{f}(U, V) \neq \emptyset,
$$

para cada vecindad $G$ de $y$ y para cualesquiera subconjuntos abiertos no vacíos $U$ y $V$ de $X$. Sean $V_{1}$ y $V_{2}$ subconjuntos abiertos en $X$ y $x_{1} \in X$, tales que:

$$
\operatorname{diám}\left(V_{1}\right)<\varepsilon \quad \mathrm{y} \quad \operatorname{diám}\left(V_{2}\right)<\varepsilon .
$$

Además,

$$
y \in V_{1} \quad \mathrm{y} \quad x_{1} \in V_{2} .
$$

Y

$$
\frac{\operatorname{diám}(X)}{2} \leq d\left(x_{1}, y\right)
$$


Por otro lado, de (26), aplicado a los conjuntos $U_{0}, V_{1}$ y $V_{2}$, se obtiene que:

$$
N_{f}\left(x, V_{1}\right) \cap N_{f}\left(U_{0}, V_{2}\right) \neq \emptyset .
$$

Luego, existe $n \in \mathbb{Z}_{+}$tal que $n \in N_{f}\left(x, V_{1}\right)$ y $n \in N_{f}\left(U_{0}, V_{2}\right)$. Esto implica que

$$
f^{n}(x) \in V_{1} \quad \text { y } \quad U_{0} \cap f^{-n}\left(V_{2}\right) \neq \emptyset .
$$

Por la Proposición 1 y de (30) se deduce que $f^{n}\left(U_{0}\right) \cap V_{2} \neq \emptyset$. Luego existe $z \in U_{0}$ tal que :

$$
f^{n}(z) \in V_{2} .
$$

De la desigualdad del triángulo se satisface que:

$$
d\left(y, x_{1}\right) \leq d\left(y, f^{n}(x)\right)+d\left(f^{n}(x), f^{n}(z)\right)+d\left(f^{n}(z), x_{1}\right) .
$$

Luego, de (27), (28) y (30), resulta que $d\left(y, f^{n}(x)\right)<\varepsilon$. Nuevamente, de (27), (28) y (31), se deduce que $d\left(f^{n}(z), x_{1}\right)<\varepsilon$. En consecuencia, de (32), se cumple que

$$
d\left(y, x_{1}\right)<\varepsilon+d\left(f^{n}(x), f^{n}(z)\right)+\varepsilon .
$$

Luego,

$$
d\left(y, x_{1}\right)-2 \varepsilon<d\left(f^{n}(x), f^{n}(z)\right) .
$$

De donde, por (29), se concluye que:

$$
\delta=\frac{\operatorname{diám}(X)}{2}-2 \varepsilon \leq d\left(x_{1}, y\right)-2 \varepsilon<d\left(f^{n}(x), f^{n}(z)\right) .
$$

Así,

$$
\delta<d\left(f^{n}(x), f^{n}(z)\right) .
$$

Por lo tanto, $(X, f)$ es sensitivo.

Como consecuencia del Ejemplo 16 y el Teorema 10, se obtiene el ejemplo siguiente.

\section{Ejemplo 17}

El sistema dinámico $([0,1], T)$ es sensitivo.

Por el Ejemplo 17 y por el Corolario 4, se obtiene el ejemplo siguiente.

\section{Ejemplo 18}

Consideremos el sistema dinámico $([0,1], T)$. Se cumple que:

1. $([0,1], T)$ es cofinitamente sensitivo.

2. $([0,1], T)$ es thickly sindéticamente sensitivo.

3. $([0,1], T)$ es multi-sensitivo.

4. $([0,1], T)$ es thick sensitivo.

En la Figura 10, en la cual se muestran algunas relaciones entre los sistemas dinámicos en la clase de los sistemas transitivos. La flecha significa que la clase de funciones precedente implica la siguiente.

De la Figura 10, se obtiene el siguiente Ejemplo 19. 


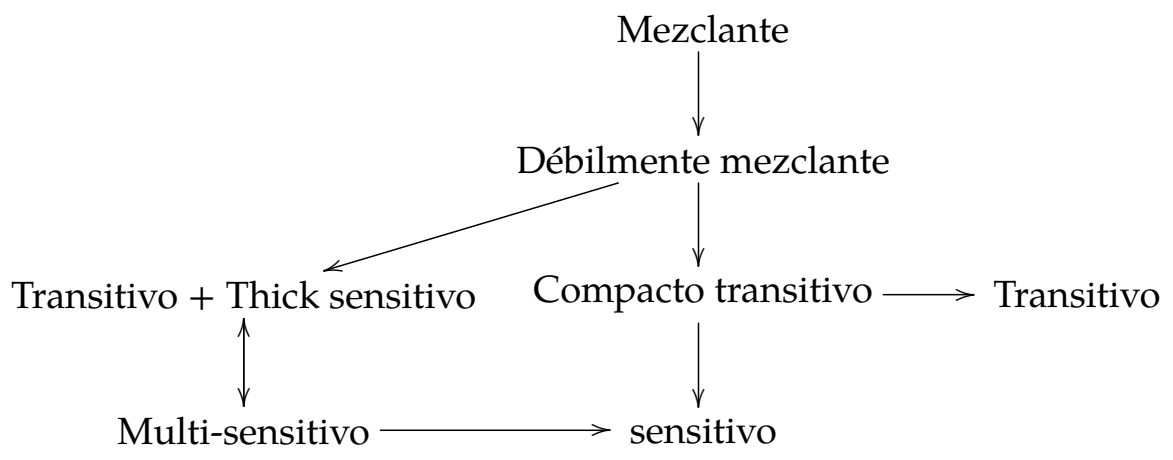

Figura 10: Diagrama que muestra las relaciones entre los sistemas dinámicos en la clase de los sistemas dinámicos transitivos.

\section{Ejemplo 19}

Consideremos el sistema dinámico $\left(X, i d_{X}\right)$, donde $i d_{X}: X \rightarrow X$ es la función identidad. Se tiene que:

1. $\left(X, i d_{X}\right)$ no es mezclante.

2. $\left(X, i d_{X}\right)$ no es débilmente mezclante.

3. $\left(X, i d_{X}\right)$ no es compacto transitivo.

Con respecto al recíproco de algunas relaciones indicadas en la Figura 10, se cumple el Teorema 11, el cual puede encontrar en [8, Corolario 3.10].

Teorema 11

Sea $(X, f)$ un sistema dinámico compacto transitivo. Si $(X, f)$ es un M-sistema, entonces $(X, f)$ es débilmente mezclante.

Finalmente, del Teorema 11 y de la Figura 10, se obtiene la Figura 11, que muestra las relaciones entre algunos tipos de sistemas dinámicos en la clase de M-sistemas. La flecha significa que la clase de funciones precedente implica la siguiente.

Compacto transitivo<smiles>[TeH]</smiles>

Débilmente mezclante $\longrightarrow$ Thick sensitivo

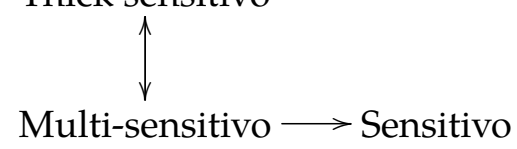

Figura 11: Diagrama que muestra las relaciones entre los sistemas dinámicos en la clase de los M-sistemas.

\section{Bibliografía}

[1] Akin E. y Kolyada S. (2014). Li-Yorke Sensitivity. Nonlinearity, 16, 1421-1433. 
[2] Bacon H. M. (1955). Differential and Integral Calculus. New York and London, McGraw-Hill.

[3] Barragán F., Santiago-Santos A., Tenorio J. F. (2016). Dynamic properties for the induced maps on n-fold symmetric product suspensions. Glasnik Matematicki Series III, 51(71), no. 2, 453-474.

[4] Barragán F., Santiago-Santos A., Tenorio J. F. (2020). Dynamic properties of the dynamical system $\left(S F_{m}^{n}(X), S F_{m}^{n}(f)\right)$. Applied General Topology, 21, n. 1, 17-34.

[5] Barragán F., Santiago-Santos A. y Tenorio J. F. (2021). Dynamic properties for the induced maps on $n$-fold symmetric product suspensions II. Topology Appl. 288, 107484.

[6] Devaney R. L. (1989). An introduction to chaotic dynamical systems. Menlo Park, California, Addison-Wesley.

[7] Glasner E. (2004). Classifying dynamical systems by their recurrence properties. Topol. Methods Nonlinear Anal., 21-40.

[8] Huang W., Khilko D., S. Kolyada, Zhang G. (2016). Dynamical compactness and sensitivity. Journal of Differential Equations, Vol. 260, 6800-6827.

[9] King Dávalos J. E. y Méndez Lango H. (2014). Sistemas dinámicos discretos. Serie: Temas de Matemáticas, Facultad de Ciencias, UNAM.

[10] Knudsen C. (1994). Chaos without nonperiodicity. American Mathematical Monthly 101, 563-565.

[11] León Torres I. (2018). Compacidad Dinámica y Sensitividad. Tesis de licenciatura, UTM.

[12] León-Torres I., Santiago-Santos A. Compacidad Dinámica y Sensitividad, enviado para su publicación, al libro Topología y sus Aplicaciones. Editores J. Angoa y otros. Dirección de fomento editorial, BUAP.

[13] Liu H., Liao L. y Wang L. (2014). Thickly syndetical sensitivity of topological dynamical system. Discrete Dyn. Nat. Soc, 583431.

[14] Rojas Carrasco, A. (2017). Nociones de Transitividad Topológica en Productos Simétricos Generalizados. Tesis de maestría, UTM.

[15] Rojas A., Barragán F., Macías S. (2020). Conceptions on topological transitivity in products and symmetric products. Turkish Journal of Mathematics, 44(2), 491-523.

[16] Ruelle D. y Takens F. (1971). On the nature of turbulence. Communications in Mathematical Physics, 167-192.

[17] Subrahmonian Moothathu, T. K. (2007). Stronger forms of sensitivity for dynamicals systems. Nonlinearity 20, 2115-2126. 